\title{
Percutaneous mitral balloon valvuloplasty. Difficult mitral valve crossing
}

\author{
Zbigniew Chmielak ${ }^{1}$, Marcin Demkow ${ }^{2}$, Jarosław Skowroński ${ }^{1}$, Paweł Tyczyński ${ }^{1}$, Dariusz Zakrzewski \\ Adam Witkowski ${ }^{1}$
}

${ }^{1}$ Department of Interventional Cardiology and Angiology, Institute of Cardiology, Warsaw, Poland ${ }^{2}$ Department of Coronary and Structural Heart Diseases, Institute of Cardiology, Warsaw, Poland ${ }^{3}$ Department of Acquired Cardiac Defects, Institute of Cardiology, Warsaw, Poland

\section{Introduction}

Percutaneous mitral balloon valvuloplasty (PMBV) is the treatment of choice for significant mitral stenosis (MS). The inability to cross the mitral valve accounts for a non-negligible rate of unsuccessful PMBV. This may be caused by unfavorable septal puncture or extensive enlargement of the left atrium (LA), both resulting in insufficient support. Advanced valvular and subvalvular degeneration may further impede valve crossing.

Surprisingly, only a few reports focus on this problem. In a study by Feldman, failure to cross the mitral valve occurred in $1.7 \%$ of cases [1]. Cribier et al. reported the occurrence of this failure at $2.6 \%$ [2].

We present a case of a successful re-attempt of PMBV done with the support of a veno-arterial loop after an unsuccessful mitral valve crossing with an Inoue balloon in the first procedure.

\section{Case report}

A 38-year-old woman with significant MS, history of ischemic stroke, and hypertension was admitted for PMBV.

In echocardiography LA enlargement to $30.0 \mathrm{~cm}^{2}$, thickening of mitral leaflets and subvalvular apparatus, fusion of posterior commissure were observed. Mitral valve area (MVA) was $0.8 \mathrm{~cm}^{2}$ by the pressure half-time (PHT) formula. Trans-mitral gradient was 21.7/10.5 mm Hg maximum and mean, respectively. The standard PMBV with the Inoue balloon was initiated via the right femoral vein. Atrial septum puncture was performed under transesophageal echocardiography (TEE) guidance. In the case of LA enlargement the right atrium is pushed to the front. So, as in the typical place of puncture the ascending aorta might be present, we usually puncture the atrial septum slightly posteriorly. Then hemodynamic measurements were made. After having placed the balloon in the LA, all of the maneuvers aiming to cross the mitral valve orifice with the balloon failed. During the second approach, a modified PMBV technique was used with a transseptal veno-arterial loop serving as a rail for the balloon's entry into the LV (Figure 1). The TEE-guided atrial septum puncture was performed again. The dedicated 6 Fr Swan-Ganz catheter, with $0.035 \mathrm{~mm}$ lumen, was introduced to the LA and next to the left ventricle (LV), through which a $300 \mathrm{~mm}$ long, 0.035 inch wire was advanced from the LA into the LV. Using the arterial access, a Multi-snare loop (PFM Medical, Germany) was advanced in a retrograde fashion through a $6 \mathrm{Fr}$-JR guide catheter into the ascending aorta and directed into the LV. Then the wire tip was caught with it and pulled out through the guide catheter and arterial sheath, completing the veno-arterial loop. The next steps of PMBV were done using the standard approach. The Inoue balloon was advanced over a 0.035 inch wire across the mitral valve and dilated to $28 \mathrm{~mm}$. The procedure was successful. Pulmonary artery pressure decreased from $105 / 32 / 65 \mathrm{~mm} \mathrm{Hg}$ to $58 / 25 / 37 \mathrm{~mm} \mathrm{Hg}$, systolic/diastolic/ mean, respectively. The fluoroscopy time was $39 \mathrm{~min}$.

Trans-mitral gradient decreased to $13 / 5.3 \mathrm{~mm} \mathrm{Hg}$ (maximum/mean) and MVA increased to $1.25 \mathrm{~cm}^{2}$ by the PHT formula and $1.6 \mathrm{~cm}^{2}$ by planimetry.

\section{Discussion}

Various techniques have been proposed to enable the anterograde trans-septal or retrograde non-transseptal approach (Stefanadis technique) for balloon entry into the LV [3].

\section{Corresponding author:}

Paweł Tyczyński MD, PhD, Department of Interventional Cardiology and Angiology, Institute of Cardiology, 42 Alpejska St, 04-628 Warsaw, Poland, phone: +48 696091 079, e-mail: medykpol@wp.pl

Received: 18.09.2017, accepted: 30.10.2017. 

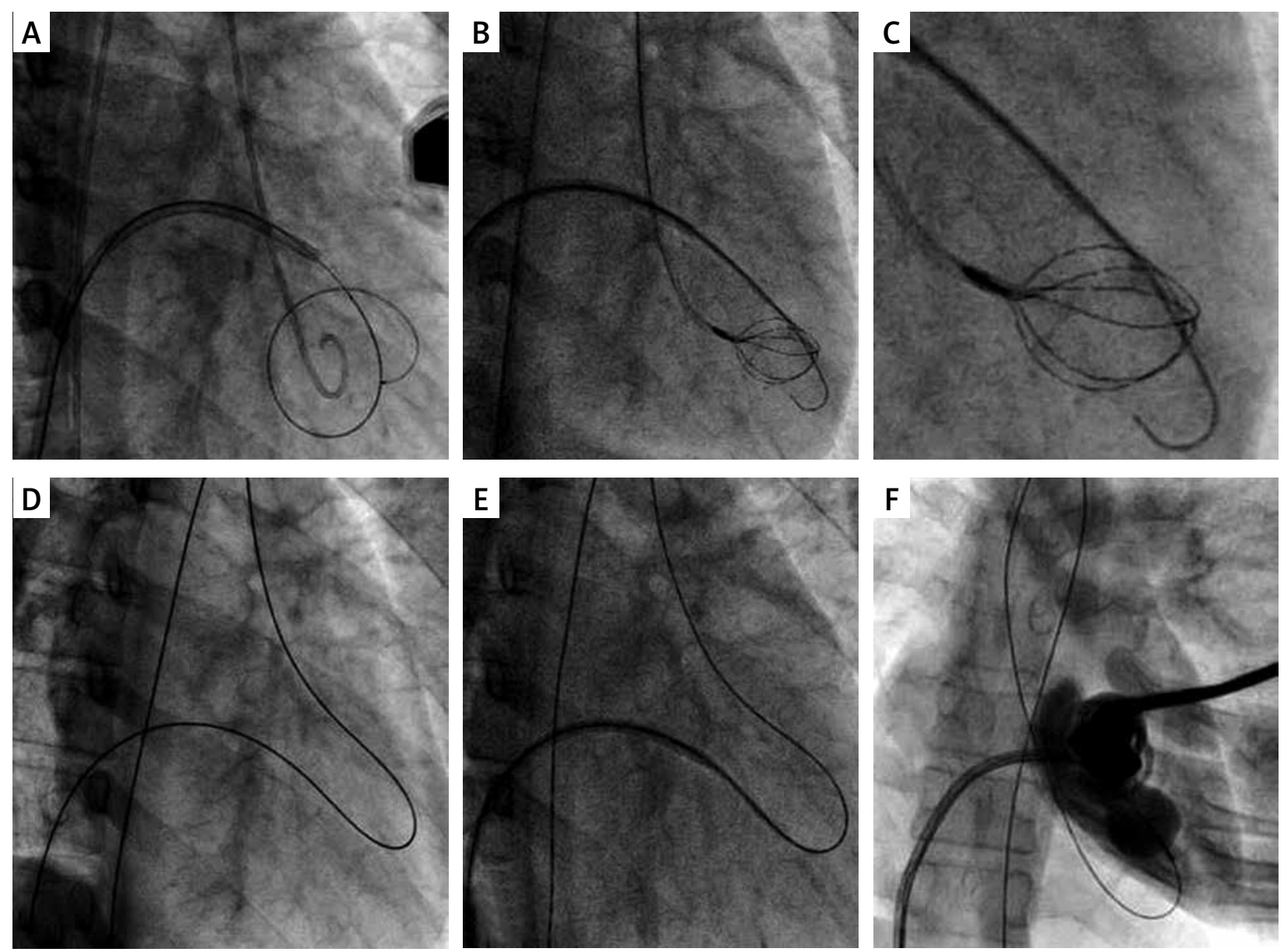

Figure 1. Procedure. A - Wire and pigtail, both in the left ventricle, $\mathbf{B}$ - catching the wire's tip with the multisnare loop, C - magnification of image B, D - veno-arterial loop, E-Inoue balloon's crossing though the mitral valve, $\mathrm{F}$ - balloon dilatation

The veno-arterial loop technique is used in different strategies such as closure of paravalvular leaks, ventricular septal defects or the transcatheter mitral valve-in-ring (TVIR) procedure, to offer better delivery support. Ateş et al. presented probably the first use of a veno-arterial loop for a challenging mitral valve crossing [4]. Technically, the wire was directed in an anterograde fashion from the LA through the LV into the ascending and descending aorta using a multipurpose catheter. Finally the loop was completed by snaring the common iliac artery and pulling it out from the sheath [4]. Thus, no multi-snare loop was used here, unlike in our case.

Recently Nanjappa et al. reported the first series of three patients with difficult mitral valve crossings, where a veno-arterial loop was created and proved to be helpful [5]. In our opinion the crossing maneuver is safe and useful, but should be done gently.

\section{Conclusions}

A veno-arterial loop may be considered a bail-out technique in cases of a problematic crossing of the mitral valve orifice during PMBV.

\section{Conflict of interest}

The authors declare no conflict of interest.

\section{References}

1. Feldman T. Hemodynamic results, clinical outcome, and complications of Inoue balloon mitral valvotomy. Cathet Cardiovasc Diagn 1994; Suppl 2: 2-7.

2. Cribier A, Eltchaninoff $H$, Koning R, et al. Percutaneous mechanical mitral commissurotomy with a newly designed metallic valvulotome: immediate results of the initial experience in 153 patients. Circulation 1999; 99: 793-9.

3. Manjunath CN, Srinivasa KH, Patil CB, et al. Balloon mitral valvuloplasty: our experience with a modified technique of crossing the mitral valve in difficult cases. Cathet Cardiovasc Diagn 1998; 44: 23-6.

4. Ateş i, Ulucan Ş, Kaya Z, et al. New technique for challenging cases of percutaneous balloon mitral valvuloplasty: the venoarterial looping. Anatol J Cardiol 2015; 15: 428-9.

5. Nanjappa V, Sadanand KS, Santhosh K, et al. Case series: difficult PTMC using novel technique of veno-arterial looping. Indian Heart J 2017; 69: 207-10. 